\title{
FOREWORD
}

\section{Establishment of Microstructural Spacing during Dendritic and Cooperative Growth}

This symposium was organized under joint sponsorship by the ASM-MSD Phase Transformations Committee and the TMS-AIME Solidification Committee to provide a forum for discussion of microstructural spacing in different morphologies during various transformations. The invited contributions were presented during the March 1983 meeting of TMS-AIME.

The size scale and phase spacing are among the most important characteristic features of regular microstructural morphologies. In practice a number of thermal and mechanical treatments have been developed to modify microstructural spacing during phase transformations. For some time there has been no generally established analysis to account for the selection of spacing under given conditions. However, recent advances in experiment have yielded accurate measurements in well-characterized systems which have stimulated new theoretical work on the analysis of the selection of spacing and scale.

Important theoretical progress has been made in applying the principle of marginal stability to the initial selection of dendrite spacing and with the description of dendrite coarsening to account for structural adjustment. Further progress in analysis and new results have been reported on spacings developed in cellular, eutectic, and monotectic structures. For solid state reactions recent experimental work on spacing selection has emphasized the importance of diffusional transport in the vicinity of the moving transformation interface. The written discussions in the papers that were available at the time of publication provide a valuable overview of recent work and point out a number of unresolved areas which should stimulate future work.

J. H. Perepezko University of Wisconsin-Madison Madison, Wisconsin

G. J. Shiflet

University of Virginia Charlottesville, Virginia 